\title{
BrkAutoDisplay: functional display of multiple exogenous proteins on the surface of Escherichia coli by using BrkA autotransporter
}

\author{
Fang Sun ${ }^{1,3 \dagger}$, Xiaoyun Pang ${ }^{1 \dagger}$, Tian Xie ${ }^{2,3}$, Yujia Zhai ${ }^{1}$, Ganggang Wang ${ }^{2^{*}}$ and Fei Sun ${ }^{*^{*}}$ (D)
}

\begin{abstract}
Background: Bacterial surface display technique enables the exogenous proteins or polypeptides displayed on the bacterial surface, while maintaining their relatively independent spatial structures and biological activities. The technique makes recombinant bacteria possess the expectant functions, subsequently, directly used for many applications. Many proteins could be used to achieve bacterial surface display, among them, autotransporter, a member of the type $V$ secretion system of gram-negative bacteria, has been extensively studied because of its modular structure and apparent simplicity. However, autotransporter has not been widely used at present due to lack of a convenient genetic vector system. With our recently characterized autotransporter BrkA (Bordetella serum-resistance killing protein A) from Bordetella pertussis, we are aiming to develop a new autotransporter-based surface display system for potential wide application.

Results: Here, we construct a bacterial surface display system named as BrkAutoDisplay, based on the structure of autotransporter BrkA. BrkAutoDisplay is a convenient system to host exogenous genes. In our test, this system is good to efficiently display various proteins on the outer membrane surface of Escherichia coli, including green fluorescent protein (GFP), various enzymes and single chain antibody. Moreover, the displayed GFP possesses green fluorescence, the enzymes CotA, EstPc and PalA exhibit catalytic activity 0.12, 6.88 and $0.32 \mathrm{mU}$ (per $5.2 \times 10^{8}$ living bacteria cells) respectively, and the single chain antibody fragment (scFv) can bind with its antigen strongly. Finally, we showed that C41(DE3) is a good strain of E. coli for the successful functionality of BrkAutoDisplay.
\end{abstract}

Conclusions: We designed a new bacterial display system called as BrkAutoDisplay and displayed various exogenous proteins on E. coli surface. Our results indicate that BrkAutoDisplay system is worthy of further study for industrial applications.

Keywords: Antibody display, Autotransporter, Bacterial surface display, Biocatalysts, BrkA

\section{Background}

Recombinant exogenous proteins, polypeptides and antibodies displayed on the surface of phage, bacteria, fungi and virus, have wide applications, such as recombinant bacterial vaccines, peptide library screening, whole cell

\footnotetext{
*Correspondence: wanggg@cib.ac.cn; feisun@ibp.ac.cn

${ }^{\dagger}$ Fang Sun and Xiaoyun Pang make equal contributions to this work ${ }^{1}$ National Laboratory of Biomacromolecules, Institute of Biophysics, Chinese Academy of Sciences, 15 Datun Road, Beijing 100101, China

${ }^{2}$ Key Laboratory of Environmental and Applied Microbiology, Chengdu Institute of Biology, Chinese Academy of Sciences, Chengdu 610041, China

Full list of author information is available at the end of the article
}

adsorption reagents, whole cell biocatalysts, whole cell solid reagents for clinical diagnosis and etc. [1-4]. In comparison to phage, fungi and virus, bacteria has been becoming a popular host for surface display application because of its simplicity of cell culture and genetic operation as well as its large capacity from small peptides to large proteins [5].

Bacterial inner membrane display system can successfully display exogenous proteins [6,7]. However, it requires the preparation of spheroplasts since the exogenous proteins are displayed on the inner membrane and not freely accessible from the bacterial outer surface. In 
contrast, for bacterial outer membrane display system that exogenous proteins are displayed on the outer membrane directly, bacterial cells can maintain their integrity and viability during application. There are many advantages of bacterial outer membrane display system. First of all, laborious and costly purification steps are avoided. Instead, the whole bacteria are collected by centrifugation and applied directly for whole-cell biocatalyst, live vaccine screening and bioremediation $[8,9]$. Secondly, the outer membrane display system can provide a genotype-phenotype link for protein libraries screening [10]. Furthermore, enzymes immobilized on the bacterial outer surface appear more stable in comparison with their free forms in solution [11].

There have been many proteins studied for displaying exogenous proteins on the bacterial outer membrane, including OmpA [12], flagellin, ice-nucleation protein (INP) [13] and autotransporters [14]. Among them, the surface display systems derived from autotransporters (ATs) of gram-negative bacteria exhibit great potentials for future wide application due to its apparent simplicity and modularity [9]. Most ATs typically act as adhesins, degradative enzymes, cytotoxins or other virulence factors $[15,16]$, including early discovered IgA protease from Neisseria [17], Ag43 from E. coli [18], AIDA from Enteropathogenic E. coli [19] and MisL from Salmonella [20]. All ATs contain an N-terminal cleavable signal peptide, a functional passenger domain and a C-terminal $\beta$-barrel domain [9]. The $\beta$-barrel domain of autotransporter is relatively uniform in size ( 300 amino acids) and assembled on the outer membrane with the aid of the $\beta$-barrel assembly machinery (BAM) complex [21-24]. After the assembly of the $\beta$-barrel domain, the self-translocation of the passenger domain starts via the hairpin model that the $\mathrm{C}$-terminal region firstly forms a hairpin in the central-pore of the $\beta$-barrel and the rest part of residues go through the pore by sliding from the $\mathrm{C}$-terminus to $\mathrm{N}$-terminus [25]. After the translocation of the passenger domain, the $\mathrm{C}$-terminal residues left in the pore of the $\beta$-barrel reach a proper position and conformation ready for cleavage in the pore $[26,27]$. Thus the $\beta$-barrel domain is, essential for passenger domain translocation across the outer membrane and is the key element for outer membrane display. ATs have been widely used as an elegant and efficient tool to display exogenous proteins and showed advantages for biotechnology and industrial (if scale up) applications [11]. However, not all target proteins can be successfully displayed [28] and till now there have been no universal rule to predict whether a target protein is suitable for AT-based surface display [9]. Furthermore, there is still lack of a convenient AT-based system for easier genetic operation and high throughput screening of target proteins.
The BrkA (Bordetella serum-resistance killing protein A) is an important virulence factor in Bordetella pertussis that confers serum resistance and mediates adherence [29]. We previously solved the crystal structure of the transmembrane $\beta$-domain of BrkA and demonstrated that the hairpin-like structure and the hydrophobic cavity at the periplasmic side of the $\beta$-domain are crucial for BrkA passenger domain translocation [30]. Besides, more interestingly, after the passenger domain is transported across the outer membrane and self-cleaved [27], the passenger domain of BrkA remains tightly associated with the bacterial surface [31]. This is a significant characteristic differing from other autotransporters. Previous extensive structural and functional studies of BrkA have enabled us to develop a BrkA-based bacterial display system, called as BrkAutoDisplay, by replacing the passenger domain region with a multi cloning site ready for various exogenous proteins insertion and secretion.

\section{Results}

\section{Construction of a vector system for BrkAutoDisplay}

The BrkA protein is translated with an $\mathrm{N}$ terminal signal peptide followed by the passenger domain and its $C$ terminal $\beta$-barrel domain (Fig. 1a). Based on our previous structural study of BrkA, we designed a new bacterial display system, which is called as BrkAutoDisplay, by replacing the passenger domain with other exogenous proteins (Fig. 1a). BrkAutoDisplay is a vector system derived from pET-22b (+) plasmid (Novagen) by inserting a synthesized DNA fragment between NdeI and Bpu1102I restriction sites (Fig. 1b). The DNA fragment (Fig. 1c) consists of the $5^{\prime}$ end nucleotides sequence encoding $\mathrm{N}$ terminal signal peptide (4-129nt), the sequence encoding a 6xHis tag (181-198nt), the multiple cloning site (MCS, 199-249nt), the sequence encoding the translocation determinants (250-810nt) [30] and the sequence encoding the $\beta$-barrel domain (811-1650nt). To be noted that, the sequence between the signal peptide and the 6xHis tag derives directly from the $\mathrm{N}$-terminal residues of BrkA and the purpose of this design is to try to avoid any potential influence of the signal peptide cleavage efficiency.

The exogenous gene can be inserted into the MCS region by molecular cloning and then the recombinant plasmid can be transformed into E. coli for protein expression and surface display assay. With the signal peptide, the translated fusion exogenous protein will be transported into the periplasmic space via a Sec-dependent pathway. After the signal peptide is cleaved, the exogenous protein can translocate across the outer membrane under the help of the $\beta$-barrel domain. Due to the characteristic of BrkA protein, the final transported exogenous protein will adhere onto the bacterial surface [30]. By 
a

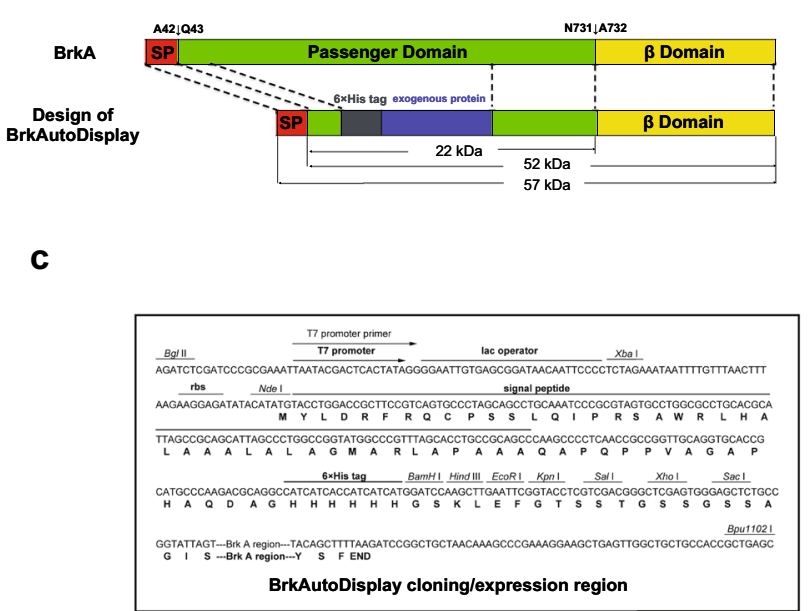

b

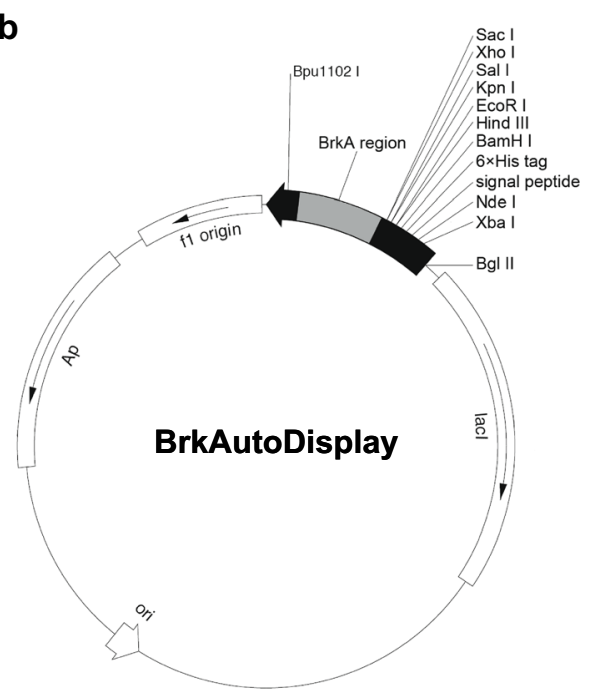

Fig. 1 The construction of the vector BrkAutoDisplay. a BrkA domain organization and the design of BrkAutoDisplay. SP, the signal peptide. The signal peptide-processing site and the passenger domain cleavage site are indicated with black arrows. All other labels are self-explanatory. $\mathbf{b}$ The vector map of BrkAutoDisplay. c The multi-clone region of the vector BrkAutoDisplay

using the BrkAutoDisplay vector, it is convenient to construct a bacterial display system for different target proteins with a single step of molecular cloning.

Furthermore, the N-terminal fused $6 x \mathrm{His}$ tag is designed to detect the expression level as well as the surface display efficiency of the target protein conveniently by western blot. Since the passenger domain of autotransporter is transported via the hairpin model that the C-terminal region firstly forms a hairpin in the central-pore of the $\beta$-barrel and the rest part of residues go through the pore by sliding from the $\mathrm{C}$-terminus to $\mathrm{N}$-terminus [25], its C-terminal residues firstly come out to the surface and thus the successful detection of the displayed $\mathrm{N}$-terminal His-tag would indicate a complete secretion of the whole target protein.

Finally, the detected surface-displayed target protein would exhibit an apparent molecular weight of $22 \mathrm{kDa}$ plus the theoretical molecular weight itself. For the uncleaved portion, the detected apparent molecular weight would be $52 \mathrm{kDa}$ plus the theoretical molecular weight of target protein (Fig. 1a).

\section{Displaying green fluorescent protein on the surface of $E$. coli}

We first tested the BrkAutoDisplay system by using the green fluorescent protein (GFP, $27 \mathrm{kDa}$ ). GFP gene was cloned into BrkAutoDisplay vector and the recombinant plasmid was named as pBAD-GFP. The pBAD-GFP was transformed into $E$. coli $\mathrm{C} 41$ (DE3), to obtain the recombinant bacteria for GFP surface display. At the same time, the empty BrkAutoDisplay vector, pBAD, was transformed into the same strain of $E$. coli as a control. The expression and surface display of GFP was assessed by fluorescence microscopy and trypsin accessibility [30]. For the experimental group, significant green fluorescence signals appear at the ends of rod-shaped bacteria (Fig. 2a), indicating a successful expression of GFP in its natural fold. Furthermore, the localization of GFP suggested that the BrkAutoDisplay system could display the target protein on the surface with a specific spatial distribution. As a control, although the cells transformed with pET-22b(+)-GFP plasmid could also express GFP successfully, green fluorescence signals appear over the whole body of the bacteria, showing no preferred spatial distribution (Fig. 2a). To be noted, BrkA has been reported to contain a sequence determinant for its localization on the pole of $E$. coli, which is relevant to the LPS (lipopolysaccharide) and membrane fluidity of $E$. coli outer membrane [32].

To further verify whether the expressed GFP has been displayed on the surface, we performed trypsin accessibility assay (see "Methods"). As shown in Fig. 2b, for pBAD-GFP construct without trypsin treatment, both the $79 \mathrm{kDa}$ full-length un-cleaved protein and the $49 \mathrm{kDa}$ cleaved transported GFP can be detected by an anti-His antibody. Treating the whole cells with trypsin resulted in the complete digestion of the $49 \mathrm{kDa}$ moiety, indicating that the transported GFP has been successfully displayed on the surface of the cell. Because it will normally take several minutes for autotransporter to complete the 
a

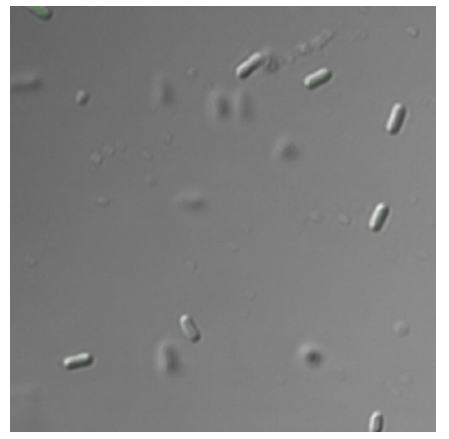

pBAD

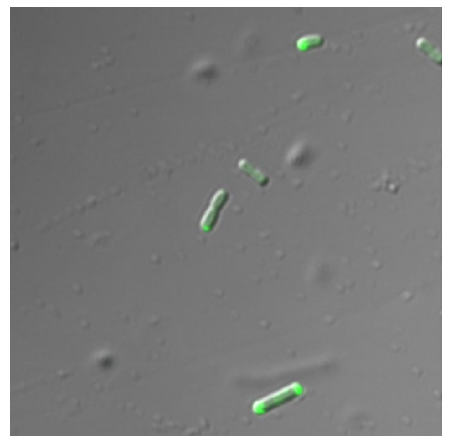

pBAD-GFP

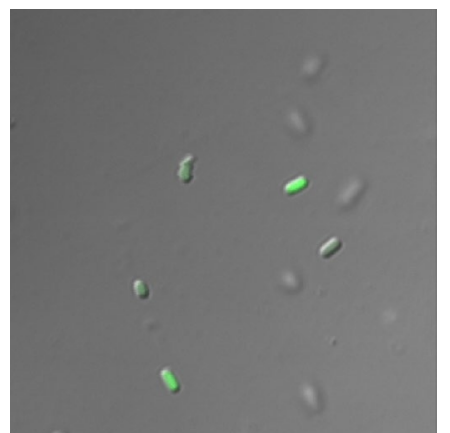

pET-22b(+)-GFP

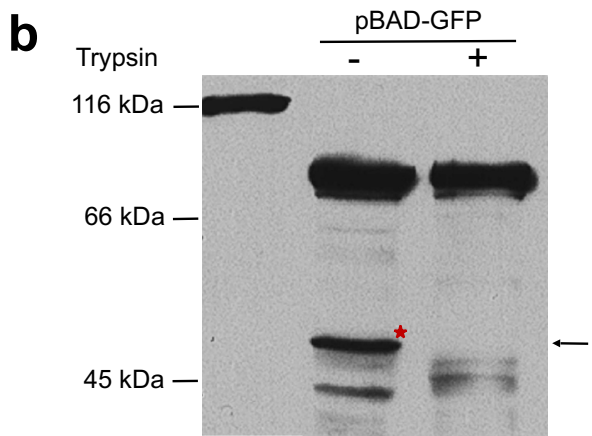

Fig. 2 Surface display of GFP by BrkAutoDisplay. a Cells transformed with empty pBAD, pBAD-GFP and pET22b(+)-GFP were observed using fluorescence microscope. $\mathbf{b}$ Trypsin accessibility assay of the displayed GFP

passenger secretion process [33], the $79 \mathrm{kDa}$ full-length protein represents the intermediates inaccessible to trypsin, which is located in the periplasm or is only targeted to the OM without passenger translocation.

\section{Displaying catalytic enzymes on the surface of $E$. coli}

The second round of test to assess the potential application of BrkAutoDisplay for whole cell biocatalysts. We selected six different enzymes to verify their surface display abilities by BrkAutoDisplay since their cDNAs are accessible, which include CotA from Bacillus subtilis (GenBank No. AIC43241.1), ECH from Caenorhabditis elegans (GenBank No. NP_491222.2), ECH-9 from Caenorhabditis elegans (GenBank No. NM_069474. 4), AtaPT from Aspergillus terreus (GenBank No. KP893683), EstPc from Psychrobacter cryohalolentis $\mathrm{K}^{\mathrm{T}}$ (GenBank No. CP000323) and PalA from Pseudomonas $s p$ (GenBank No. AY055203). CotA (MW $58 \mathrm{kDa}$ ) is a classical laccase, catalyzing the reduction of oxygen to water accompanied by the oxidation of polyphenol compounds. The laccase has been widely applied into textile industry, food engineering, medicine in organic synthesis and bio-remediation of contaminated environment
[34]. ECH and ECH-9 are key enzymes for the cellular $\beta$-oxidation cycle and responsible for the second and third steps of reactions respectively [35, 36]. The ECH (MW $29 \mathrm{kDa}$ ) is an enoyl-CoA hydratase and adds a hydroxyl group and a proton to the unsaturated $\beta$-carbon of a fatty-acyl CoA and the ECH-9 (MW $49 \mathrm{kDa}$ ) is a L-3-hydroxyacyl-CoA dehydrogenase and oxidizes the hydroxyl group of L-3-hydroxyacyl-CoA to a keto group. AtaPT (MW $46 \mathrm{kDa}$ ) is an aromatic prenyltransferase and responsible for the biosynthesis of diverse active prenylated aromatics [37]. The EstPc (MW $33 \mathrm{kDa})$ is a cold-active esterase that exhibits high activity at low temperature $\left(15-25^{\circ} \mathrm{C}\right)$ to catalytic hydrolysis of p-nitrophenyl butyrate [38]. The PalA (MW $38 \mathrm{kDa}$ ) is a lipolytic enzyme that hydrolyzes triglycerides into glycerol and free fatty acids [39].

The DNAs of CotA, ECH-9, AtaPT, ECH, EstPc and PalA were inserted into BrkAutoDisplay vector and then transformed into E. coli C41(DE3) to get six constructs, pBAD-CotA, pBAD-ECH-9, pBAD- AtaPT,pBAD-ECH, pBAD-EstPc and pBAD-PalA respectively. The expected molecular weights of the displayed targets are 81, 71, $68,51,55$ and $60 \mathrm{kDa}$ respectively. Treating the whole 
cells with trypsin resulted in the complete digestion of the corresponding moieties, indicating that all the six enzymes have been successfully displayed on the surface of the cell (Fig. 3a).

Beside trypsin accessibility assay, we further used flow cytometry to quantify the display efficiencies of those six enzymes (Fig. 3b). All the cells were treated with the anti-His antibody followed by FITC conjunct goat antimouse secondary antibody. Subsequently, the samples were investigated by flow cytometer. Only the successfully displayed enzyme with $6 \mathrm{x}$-His tag in N-terminus could be labeled by FITC. As a result, the quantified display efficiencies on the surface of $E$. coli for pBAD-CotA, pBAD-ECH-9, pBAD-AtaPT, pBAD-ECH, pBAD-EstPc and pBAD-PalA are 30.7, 9.2, 51.8, 64.2, 42.3 and $55.8 \%$ respectively, in comparison to the background value of $0.4 \%$ for the control group (Fig. 3b). Both trypsin accessibility assay and flow cytometry data suggest successful displays of all six enzymes although variations of display efficiencies exist.

With the catalytic assay systems available for CotA, EstPc and PalA, we tested the catalytic activities of these
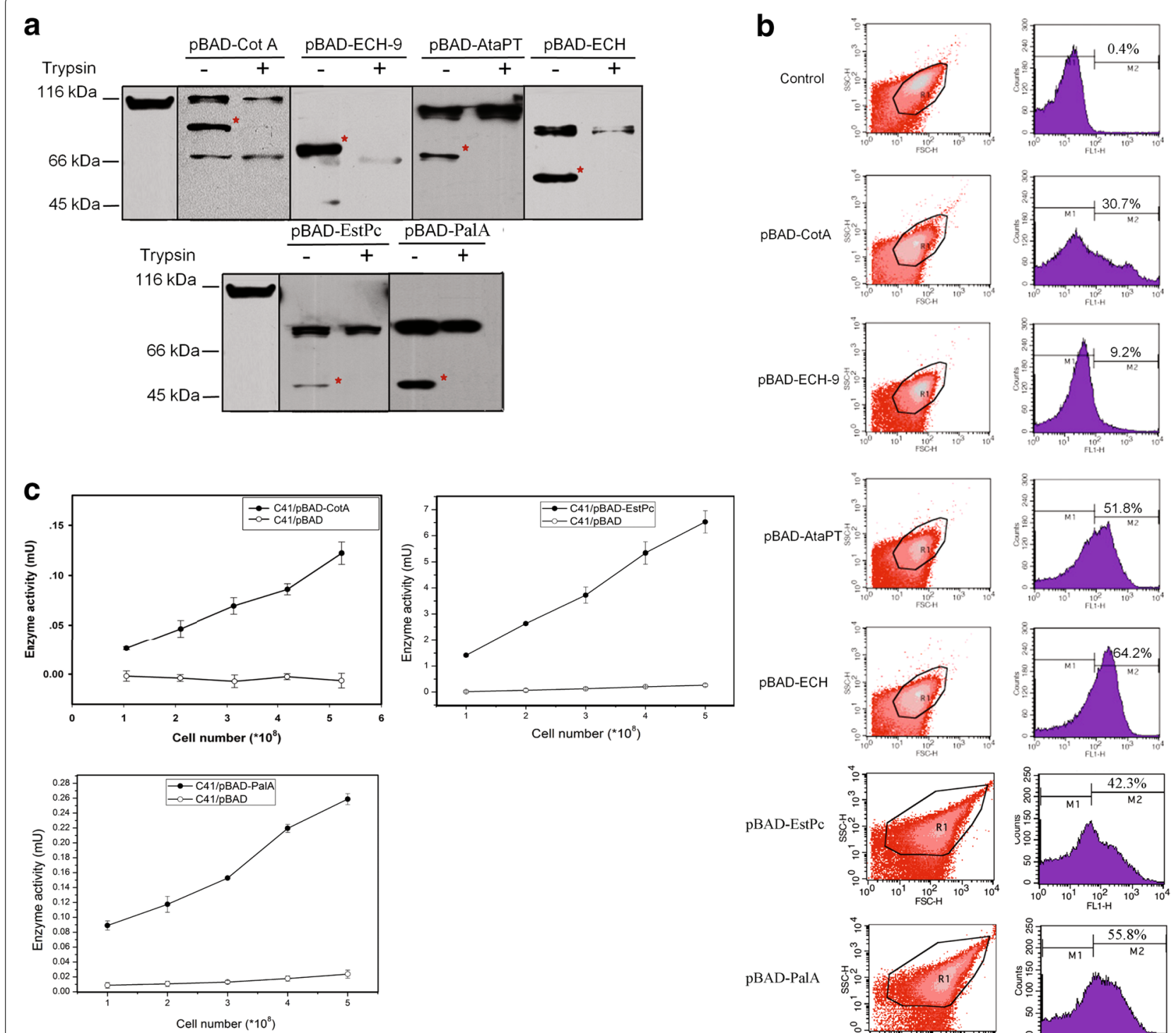

pBAD-EstPc
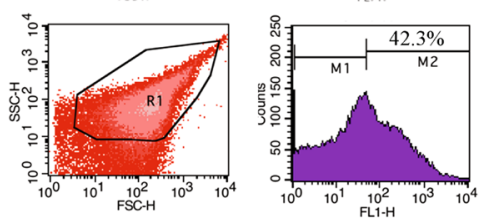

pBAD-PalA
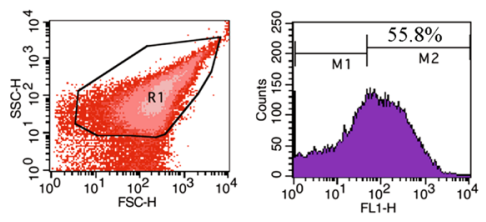

Fig. 3 Surface display of enzymes. a Trypsin accessibility assay of the displayed enzymes. b Surface display efficiency quantification by flow cytometry. c Enzymatic activity assays of the cells with displayed enzymes (CotA, EstPc and PalA) and the control group with empty pBAD. The enzymatic activities were calculated and calibrated (see "Methods") 
displayed enzymes to further verify the ability of BrkAutoDisplay for the application of whole cell biocatalysts. As shown in Fig. 3c, the recombinant pBAD-CotA cells possess the laccase activity by oxidizing the substrates 2,2'-Azino-bis (3-ethylbenzothiazoline-6-sulfonic acid) diammonium salt (ABTS). The whole cell enzyme activity increases with the increase of the number of cells, and per $5.2 \times 10^{8}$ bacteria cells can reach the activity of $0.12 \mathrm{mU}$ CotA. As a control, the cells that were transformed with empty BrkAutoDisplay plasmid showed no laccase activity (Fig. 3c).

For the enzymatic activity assays of the recombinant pBAD-EstPc and pBAD-PalA cells, experiments were conducted at $25{ }^{\circ} \mathrm{C}$ by using p-nitrophenyl butyrate (C4) as the substrate [38]. The whole cell enzyme activity increases with the increase of the number of cells, and per $5.2 \times 10^{8}$ bacteria cells can reach the activity of 6.88 and $0.32 \mathrm{mU}$ for EstPc and PalA respectively. As a control, the cells transformed with empty BrkAutoDisplay plasmid showed no enzymatic activity (Fig. 3c).

As a result, the three kinds of bacteria here harvesting the corresponding recombinant constructs show significant catalytic activities, which can be improved for the future industrial applications.

\section{Displaying single chain antibody and comparison with Ag43 AutoDisplay system}

Another important application of surface display is antibody presentation [40]. Here we selected a single chain antibody, ATscFv (MW $28 \mathrm{kDa}$ ), to assess the ability of BrkAutoDisplay to display an active antibody. ATscFv was cloned from Prof. Jie Tang's lab (IBP, CAS) and it can recognize the antigen human tumor necrosis factor alpha (hTNF $\alpha$ ). Xiao et al. has used the Ag43-based AutoDisplay system to display the active ATscFv successfully by comparing with the INP-based surface display system [41]. In the present study, we will further compare the display abilities and efficiencies of ATscFv between Ag43based system (Ag43ß) and our BrkAutoDisplay system.

The ATscFv gene was inserted into the BrkAutoDisplay plasmid with the name of pBAD-ATscFv. Then the recombinant plasmids, $\mathrm{pBAD}-\mathrm{ATscFv}$ and $\mathrm{Ag} 43 \beta$-ATscFv (a gift from Prof. Hai-Ying Hang), were transformed to E. coli C41(DE3). The expected molecular weights of the displayed $\mathrm{ATscFv}$ are 50 and $64 \mathrm{kDa}$, respectively. Trypsin accessibility assay revealed that $\mathrm{ATscFv}$ can be significantly displayed on the bacterial surface for pBAD$\mathrm{ATscFv}$ while less efficient for Ag43ß-ATscFv (Fig. 4a).

The display efficiencies of pBAD-ATscFv and Ag43 $\beta$ $\mathrm{ATscFv}$ were further quantified via cell cytometry approach. All the cells were treated with the anti-His antibody followed by FITC conjunct goat anti-mouse secondary antibody and then investigated by flow cytometer.
Only the successfully displayed enzyme with its $\mathrm{N}$-terminal $6 \mathrm{x}$-His tag could be labeled by FITC. As a result, the quantified display efficiencies of ATscFv for pBADATscFv and Ag43ß-ATscFv are 76.1 and $68.2 \%$, respectively, in comparison to the background value of $0.57 \%$ for the control group (Fig. 4b).

To detect antigen-binding ability of displayed AtscFv, the liquid cells were incubated with the antigen GFPhTNF $\alpha$ and thereafter examined via flow cytometry (Fig. 4c). As a result, the quantified antigen-binding efficiencies for the cells of pBAD-ATscFv and Ag43ßATscFv are 47.2 and $29.7 \%$, respectively, compared with the background value of $2.4 \%$ (Fig. 4c). The less antigenbinding efficiency (Fig. 4c) in comparison to the surface display efficiency (Fig. 4b) suggests that there are portions of displayed ATscFv lack of antigen binding ability, which might be due to the failure of correct folding, the potential spatial obstacle or other unknown reasons.

All of above, both Ag43-based system and BrkAutoDisplay system can successfully display single chain antibody ATscFv on the surface of the cell with significant antigen binding activity, while, the current data suggested that BrkAutoDisplay system played a better performance than Ag43-based system in either antibody display efficiency or antigen binding activity.

\section{Display abilities of BrkAutodisplay by different bacterial strains}

The above bacterial surface display assays were performed by using the strain of E. coli C41 (DE3). To investigate whether the exogenous protein display by BrkAutoDisplay system is strain-dependent, we transformed the above recombinant plasmids into different strains of E. coli Bl21 (DE3) and E. coli Rosetta (DE3) and assessed the surface display abilities of those trains. After induction of the cells, the target protein expression levels were examined by western blot using anti-His antibody (Fig. 5). However, only the full-length un-cleaved moieties were observed for GFP, enzymes and ATscFv, suggesting a failure of passenger translocation onto the outer membrane [30]. Therefore, BrkAutoDisplay system is strain-dependent and does not work with the strain of $E$. coli Bl21 (DE3) and Rosetta (DE3).

\section{Discussions}

In the present work, we designed a new autotransporter mediated bacterial surface display system BrkAutoDisplay based on the structure of BrkA. The system includes an easy-to-use plasmid with a multi-clone site and a workflow of assessing the surface display ability and efficiency, which enables other researchers to use this system easily via simple genetic operation and detect the efficiency of surface display via standard antibody and 


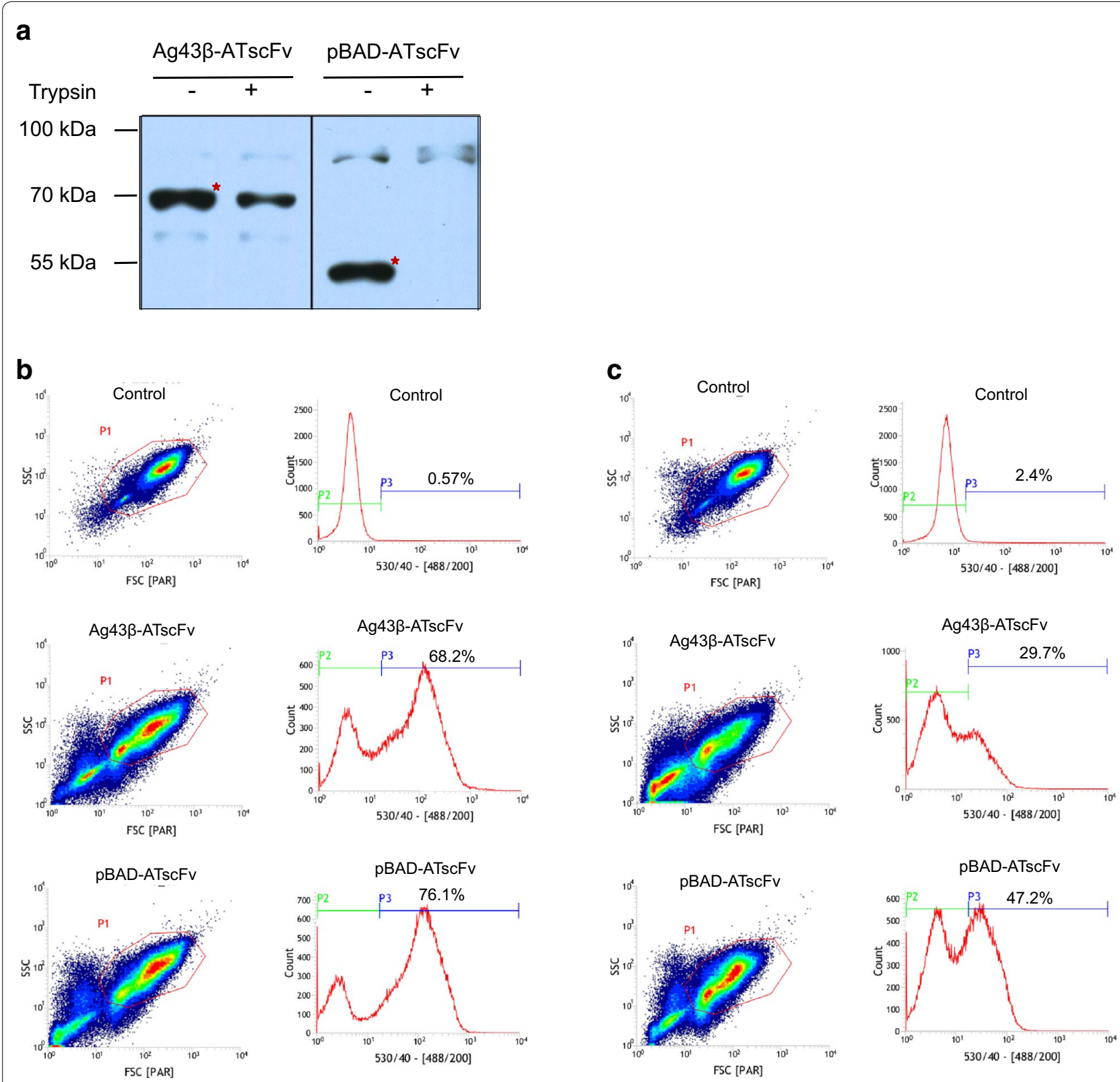

Fig. 4 Surface display of ATscFv and comparison with Ag43-based surface display system. a Trypsin accessibility assay of the displayed ATscFv. b Surface display efficiency quantification by flow cytometry. c Antigen binding ability of displayed ATscFv quantified via flow cytometry

routine western blot assay. The unique characteristic of BrkA is that the cleaved passenger domain remains adhered on the bacterial surface, which is conducive to keep the displayed exogenous proteins associated onto the cell, thus, the functionalized bacteria can be recycled/ re-used conveniently. Three kinds of exogenous proteins including GFP, enzymes and antibodies have been tested using this new bacterial surface display system, showing its advantages of the wide applicability, large capacity and high efficiency.
The successful display of GFP by BrkAutoDisplay system suggests that this system is applicable to display those proteins with $\beta$-barrel structural motif. Furthermore, in the future, the gene of GFP could be incorporated into the BrkAutoDisplay plasmid to enable the surface display of a GFP-fused target protein, which will make the assessment of the display ability and efficiency even easier via fluorescence signal and fluorescence microscopy.

Enzymes immobilized on surfaces appeared to be more stable compared to free molecules. Using BrkAutoDisplay 


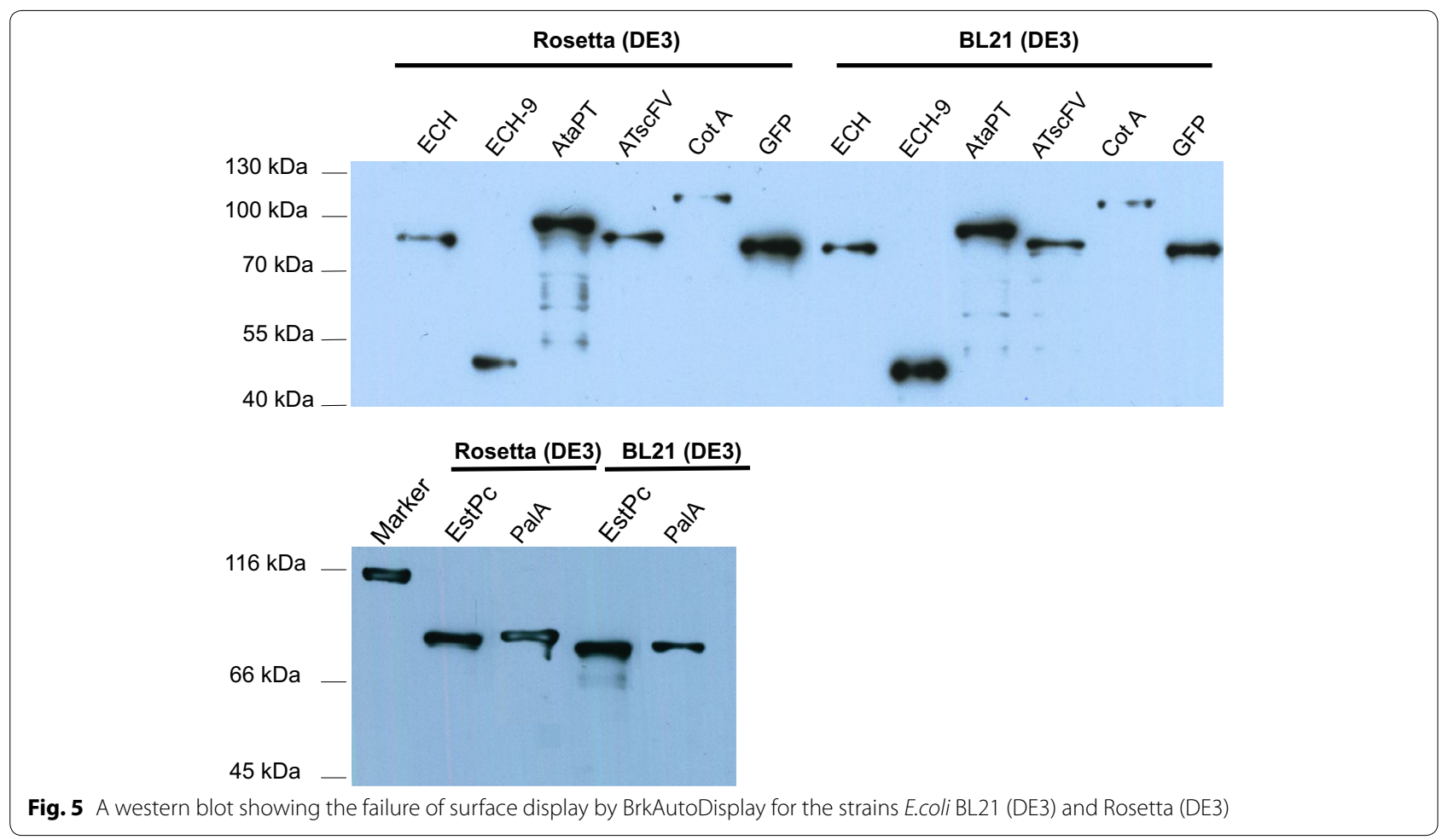

system, it is possible by a standard protocol to decorate bacterial cell surfaces with target enzymes and produce large amount of these cells with a minimum of costs and equipment. Moreover, these cells can be recovered and reused in several subsequent process cycles [11]. The E. coli cells decorated with CotA, EstPc and PalA via BrkAutoDisplay system showed the significant laccase, esterase and lipolytic activities $(0.12,6.88$ and $0.32 \mathrm{mUs}$ per $5.2 \times 10^{8}$ living bacterial cells) respectively, which would have wide applications in industry and environment bioremediation.

Moreover, for some enzymes, their activities need the presence of their chaperones. To display those enzymes on bacterial surfaces in their functional state, it is possible to co-express and co-display both the enzyme and its chaperone on the surface, e.g. the co-expression of the lipase and its chaperone foldase [2]. For this purpose, BrkAutoDisplay system can be further adapted to yield two plasmids containing different antibiotics genes and different copy operons, enabling co-expression of different target proteins.

Recombinant antibodies are increasingly used in many applications such as clinical diagnosis and therapeutics [40], which require antibodies with high antigen affinity and specificity [4]. Antibody surface display technology is critical for novel antibody screening and antibody affinity maturation. In the previous researches, Ag43 $\beta$ has been applied to present different vaccine candidates [9, 42,
43]. In the present work using the antibody of ATscFv, we found that BrkAutoDisplay has a superior performance than Ag43 $\beta$, suggesting a potential application of BrkAutoDisplay for the future antibody development.

In the present work, we tested the strain-dependent display ability of BrkAutoDisplay found that only $E$. coli C41 (DE3) can work well in comparison to E. coli BL21 (DE3) and Rosetta (DE3). Since the overexpression of exogenous gene is normally toxic to the host, E. coli C41 (DE3) might have a better control of expression level of exogenous gene, which has enabled this strain to express many toxic membrane proteins, and might have additional mechanisms to help exogenous autotransporters to target onto the outer membrane, which is essential for the surface display of the exogenous proteins.

\section{Conclusion}

BrkAutoDisplay is a new bacterial surface display system, which includes an easy-to-use plasmid with a multi-clone site and a workflow of assessing the surface display ability and efficiency. BrkAutoDisplay enables researchers to use this system easily via simple genetic operation and detect the efficiency of surface display via standard antibody and routine western blot assay. BrkAutoDisplay exhibits potencies of wide applications for whole-cell biocatalysts, environment remediation and antibody development. In the future, more work, such as additional testimonies using industrial enzymes and medical antibodies, 
stability test of the displayed target and scalable ability test of the system, will be performed.

\section{Methods}

\section{Construction of the vector BrkAutoDisplay}

The vector BrkAutoDisplay is derived from the vector pET-22b(+) (Merck Novagen) by inserting a synthesized DNA fragment (see RESULT) between the restrict digestion sites NdeI and Bpu1102 I. The DNA fragment was synthesized via the service from GeneWiz. The nucleotides from 130 to 180 of the fragment encode a peptide sequence corresponding the residues of BrkA from $\mathrm{Glu}^{43}$ to $\mathrm{Gly}^{59}$. The nucleotides from 250 to 1650 of the fragment encode a peptide sequence corresponding the residues of BrkA from $\mathrm{Ala}^{545}$ to Phe ${ }^{1013}$.

\section{Molecular cloning and surface display}

The genes encoding GFP, CotA, ECH-9, AtaPT, ECH, EstPc, PalA and ATscFv were amplified by polymerase chain reaction (PCR) using the primer set in Table 1. The genes of ECH-9 and ECH were amplified from the cDNA library of C. Elegans. The gene of ATscFv is a kind gift from Prof. Hai-Ying HANG (Institute of Biophysics, Chinese Academy of Sciences, Beijing). The gene of AtaPT is a kind gift from Prof. Jungui Dai (Institute of Materia-Medica, Peking Union Medical College and Chinese Academy of Medical Sciences). The genes of EstPc and PalA were synthesized via the service from GeneWiz. All of the PCR amplified fragments were digested with the restriction endonucleases BamH I (or EcoRI) and Kpn I (or XhoI) and then linked to the BrkAutoDisplay plasmid that was pre-treated with BamH I (or EcoRI) and Kpn I (or XhoI), yielding the recombinant plasmids, pBADGFP, pBAD-CotA, pBAD-ECH-9, pBAD-AtaPT, pBAD$\mathrm{ECH}, \mathrm{pBAD}-\mathrm{EstPc}, \mathrm{pBAD}-\mathrm{PalA}$ and pBAD-ATscFv. The recombinant plasmids were verified by sequencing, and then were transformed to E. coli strains C41 (DE3), BL21 (DE3) and Rosetta (DE3), respectively for protein expression. The empty BrkAutoDisplay plasmid was also transformed as a control. In addition, the recombinant plasmid ATscFv-Ag433 in pET-22b (+), a kind gift from Prof. Hai-Ying HANG, was transformed to E. coli strains C41 (DE3). The transformed cells were grown at $37{ }^{\circ} \mathrm{C}$ in Lysogeny Broth (LB) medium supplemented with ampicillin $(100 \mu \mathrm{g} / \mathrm{ml})$ until the $\mathrm{OD}_{600}$ reached approximately 0.8-1.0. Then the recombinant protein expression was induced by the addition of $0.2 \mathrm{mM}$ IPTG (isopropyl $\beta$-D1-thiogalactopyranoside) for $16 \mathrm{~h}$ at $16^{\circ} \mathrm{C}$.

\section{Trypsin accessibility assay and western blot analysis}

After induction for $16 \mathrm{~h}, 1 \mathrm{ml}$ of culture was harvested by centrifugation at $2000 \mathrm{~g}, 4{ }^{\circ} \mathrm{C}$ for $10 \mathrm{~min}$ and re-suspended in $200 \mu \mathrm{l}$ of PBS. For $200 \mu \mathrm{l}$ of cells, $4 \mu \mathrm{l}$ of $10 \mathrm{mg} / \mathrm{ml}$ trypsin (Merck, 108367) was added. After incubation at $37{ }^{\circ} \mathrm{C}$ for $10 \mathrm{~min}$, the cells were put on ice immediately and $20 \mu \mathrm{l}$ of FBS (fetal bovine serum) was added to stop digestion. The cells were harvested, washed three times with PBS containing $10 \%$ FBS and once in PBS alone. The washed cells were finally re-suspended in $5 \%$ SDS loading buffer and boiled for $20 \mathrm{~min}$.

Table 1 Primers for the target proteins cloning

\begin{tabular}{|c|c|c|c|}
\hline Primer name & Sequence & $\begin{array}{l}\text { Target protein } \\
\text { to be displayed }\end{array}$ & Restriction sites \\
\hline F1 & 5'-CGGGATCCATGGTGAGCAAGGGCGAG-3' & GFP & BamHI and Xhol \\
\hline R1 & 5'-CCGCTCGAGCCCTTGTACAGCTCGTCCA-3' & & \\
\hline F2 & 5'-CGGGATCCATGACACTTGAAAAATTTGTGG-' & CotA & BamHI and Xhol \\
\hline R2 & 5'-CCGCTCGAGCCTTTATGGGGATCAGTTA-3' & & \\
\hline F3 & 5'-CGGGATCCATGGGCAAAGTTCCAGAAGAAG-3' & $\mathrm{ECH}-9$ & BamHI and Kpnl \\
\hline R3 & 5'-GGGGTACCTAGTTTTGATGACATCAGTTTG-3' & & \\
\hline F4 & 5'-CGGGATCCCGGCCCTGGCAGATCCTGA-3' & AtaPT & BamHI and Kpnl \\
\hline R4 & 5'-GGGGTACCCACACGTGCGACATTTCCCGCAA-3' & & \\
\hline F5 & 5'-CGGGATCCATGTCTGGAAAAGTGGTTAG-3' & $\mathrm{ECH}$ & BamHI and Kpnl \\
\hline R5 & 5'-GGGGTACCTTTCTTGACGACCGCTTCGAG-3' & & \\
\hline F6 & 5'-CGGAATTCATAAATACCACCCA AAAGATTATTC-3' & EstPc & EcoRI and Kpnl \\
\hline R6 & 5'-GGGGTACCGTTCTTTAACCCTTCACGAAACGC-3' & & \\
\hline F7 & 5'-CGGAATTCATGATCAAAC AGACGTTGTTTGTACC-3' & PalA & EcoRl and Kpnl \\
\hline R7 & 5'-GGGGTACCTTCGTCCTGA TGAGCGCGCA ACGT-3' & & \\
\hline F8 & 5'-CGGAATTCATGGATATCGGAATTAATTCGGATCC-3' & ATscFv & EcoRl and Xhol \\
\hline R8 & 5'-CCGCTCGAGCCCCGTTTTATTTTCCAACTTTG-3' & & \\
\hline
\end{tabular}


As a control, another $200 \mu \mathrm{l}$ aliquot of cells was simultaneously processed in the same manner without added trypsin. Samples were resolved by SDS/PAGE (12\% gels) and transferred on to nitrocellulose membranes (Millipore) for $70 \mathrm{~min}$ at $300 \mathrm{~mA}$ at $4{ }^{\circ} \mathrm{C}$. The non-secreted and secreted proteins were detected by anti-His antibody (Sigma) and horseradish peroxidase-conjugated goat anti-mouse secondary antibody (ZSGB-Bio) with a working dilution of 1:3000 and 1:5000 respectively. The membranes were incubated in luminal reagent (Millipore) and exposed to film.

\section{Fluorescence assay}

The $200 \mu \mathrm{l}$ culture was harvested and washed with $200 \mu \mathrm{l}$ of PBS. Then $7 \mu \mathrm{l}$ of samples were dipped onto a microscope slide, which was observed by confocal laser scanning microscope OLYMPUS FV1000.

\section{Assay with flow cytometry}

The cells were incubated with anti-His antibody (1:3000 in PBS containing $1 \% \mathrm{BSA}$ ) for $1 \mathrm{~h}$ at room temperature. Then the cells were washed again with PBS containing $1 \%$ BSA, and incubated with FITC conjunct goat antimouse antibody (Thermo Scientific, Immuno Research, 1:100 in PBS containing $1 \% \mathrm{BSA}$ ) for $1 \mathrm{~h}$ at room temperature in dark. After labeling, cells were washed once with PBS containing $1 \%$ BSA and re-suspended in $1 \mathrm{ml}$ of PBS containing $1 \%$ BSA. The fluorescence associated with the cells were detected using FACS Influx cell sorter (BD Biosciences).

To detect the antigen-binding ability of the displayed ATscFv, the $1 \mathrm{ml}$ culture was harvested and washed with PBS. Then the cells were incubated with GFP-hTNF $\alpha$ ( $2 \mathrm{mg} / \mathrm{L}$ in PBS containing $1 \% \mathrm{BSA}$ ) for $1 \mathrm{~h}$ at room temperature in dark. After labeling, cells were washed once with PBS containing $1 \%$ BSA and re-suspended in $1 \mathrm{ml}$ of PBS containing $1 \%$ BSA. The fluorescence associated with the cells was detected and sorted via FACS Influx cell sorter (BD Biosciences).

\section{Laccase activity assay of displayed CotA}

The laccase activity was detected by monitoring the oxidation of 2,2'-Azino-bis (3-ethylbenzothiazoline6-sulfonic acid) diammonium salt (ABTS, Sigma). The unit (1U) of laccase activity is defined as the amount of enzyme required for converting $1.0 \mu \mathrm{mol}$ substrates in a minute under the condition of $40{ }^{\circ} \mathrm{C}$ and $\mathrm{pH}$ 5.0.

The culture was harvested and washed with the same step above. $50 \mu \mathrm{l}$ of bacteria suspension, $900 \mu \mathrm{l}$ of sodium citrate-Sodium dihydrogen phosphate buffer ( $\mathrm{pH} 5.0$, $100 \mathrm{mM})$ and $50 \mu \mathrm{l}$ of ABTS $(1 \mathrm{mM})$ aqueous solution were mixed. The mixture was incubated in $40{ }^{\circ} \mathrm{C}$ water for $3 \mathrm{~min}$. The optical absorbance at $420 \mathrm{~nm}$ was monitored. The laccase activity can be calculated using the formula,

$$
\frac{\Delta O D_{420} \times V \times 1000}{\mathrm{t} \times \varepsilon_{A B T S} \times \mathrm{d}}
$$

where, $\triangle O D_{420}$ is the change of the optical absorbance at $420 \mathrm{~nm}, \mathrm{t}$ represents the reaction time $(\mathrm{min}), \mathrm{V}$ is the volume of the reaction system $(\mathrm{ml}), \varepsilon_{A B T S}$ is the molar absorption coefficient of ABTS at $420 \mathrm{~nm}(36 \mu \mathrm{mol} /$ $\mathrm{ml} \times \mathrm{cm})$ and $\mathrm{d}$ is the optical path $(\mathrm{cm})$.

\section{Esterase activity assay of displayed EstPc}

Esterase activity assays were performed using p-nitrophenyl butyrate $(\mathrm{C} 4)$ as an ester substrate (Sigma). The reaction mixture contained $5 \mu \mathrm{l}$ of a substrate $(10 \mathrm{mM}$ ester in 2-propanol), $190 \mu \mathrm{l}$ buffer $(20 \mathrm{mM}$ Tris- $\mathrm{HCl}$, $0.1 \mathrm{M} \mathrm{NaCl}, \mathrm{pH} 9.0$ ) and $5 \mu \mathrm{l}$ of PBS washed intact cells. The absorbance at $415 \mathrm{~nm}$ was determined at $25{ }^{\circ} \mathrm{C}$ for 30 min with Multiskan (Thermo). One unit of activity was defined as the release of $1 \mu \mathrm{mol}$ of $\mathrm{p}$-nitrophenol per minute [38]. The esterase activity can be calculated using the following formula,

$$
\frac{\Delta O D_{415} \times V \times 1000}{\mathrm{t} \times \varepsilon_{4-\text { pnitrophenyl }} \times \mathrm{d}}
$$

where, $\varepsilon_{\mathrm{p}-\text { nitrophenyl }}$ is the molar absorption coefficient of p-nitrophenol at $415 \mathrm{~nm}(18 \mu \mathrm{mol} / \mathrm{ml} \times \mathrm{cm})$.

\section{Lipolytic activity assay of displayed PalA}

For the lipolytic activity assay of PalA, $5 \mu \mathrm{l}$ of $10 \mathrm{mM}$ p-nitrophenyl butyrate (Sigma) was added to $195 \mu \mathrm{l}$ of PBS ( $\mathrm{pH} 7.4$ ) with re-suspended cultured cells. The absorbance at $415 \mathrm{~nm}$ was determined at $25{ }^{\circ} \mathrm{C}$ for 30 min with Multiskan (Thermo). The lipolytic activity of PalA can be calculated in a same way with EstPc above.

\section{Abbreviations}

ABTS: 2,2'-Azino-bis (3-ethylbenzothiazoline-6-sulfonic acid) diammonium salt; Ag43: antigen 43; AIDA: adhesin involved in diffuse adherence; AtaPT: aromatic prenyltransferase; ATscFv: single chain antibody fragment; EstPc: esterase from Psychrobacter cryohalolentis K5 ${ }^{\top}$; PalA: Pseudomonas sp. autotransporter lipolytic protein; PBAD: plasmid BrkAutoDisplay; BrkA: Bordetella serum-resistance killing protein A; CotA: spore coat protein (laccase); $\mathrm{ECH}$ : enoyl-CoA hydratase; ECH-9: L-3-hydroxyacyl-CoA dehydrogenase; FBS: fetal bovine serum; FITC: fluorescein isothiocyanate; GFP: green fluorescent protein; IgA: immunoglobulin A; INP: ice-nucleation protein; IPTG: isopropyl $\beta$-D-1thiogalactopyranoside; LB: lysogeny Broth medium; LPS: lipopolysaccharide; MisL: protein of membrane insertion and secretion; MCS: multiple cloning site; OmpA: outer membrane protein A; OM: outer membrane protein; PAGE: polyacrylamide gel electrophoresis; PBS: phosphate buffered saline; PCR: polymerase chain reaction; SDS: sodium dodecyl sulfonate; hTNF $\alpha$ : antigen human tumor necrosis factor alpha.

\section{Authors' contributions}

FS initiated and supervised the project. $X P$ and $Y Z$ designed the vector of BrkAutoDispaly. XP performed the display assay of GFP. FS performed the display assay of enzymes and antibodies. TX and GW performed the enzymatic 
assay of displayed CotA. FS, FS and GW wrote the manuscript. All authors read and approved the final manuscript.

\begin{abstract}
Author details
${ }^{1}$ National Laboratory of Biomacromolecules, Institute of Biophysics, Chinese Academy of Sciences, 15 Datun Road, Beijing 100101, China. ${ }^{2}$ Key Laboratory of Environmental and Applied Microbiology, Chengdu Institute of Biology, Chinese Academy of Sciences, Chengdu 610041, China. ${ }^{3}$ University of the Chinese Academy of Sciences, Beijing 100049, China.
\end{abstract}

\section{Acknowledgements}

This work was supported by grants from the National Basic Research Program (973 Program) of Ministry of Science and Technology of China (2011CB910301 and 2014CB910700), and the grant from National Natural Science Foundation of China (30970569 and 21261130090). We would like to thank Prof. Hai-Ying Hang for his kindness to provide the Ag43 AutoDisplay system, the cDNAs of ATsCFv antibody and the constructs for expressing GFP-hTNF $\alpha$. We would like to give our thanks to Dr. Yan Teng from Center of Biological Imaging, Core Facility for Protein Sciences, Institute of Biophysics, Chinese Academy of Sciences for her help on confocal imaging and Dr. Junying Jia from Lab of Structural and Functional Analysis, Core Facility for Protein Sciences, Institute of Biophysics, Chinese Academy of Sciences for his help on flow cytometry.

\section{Compliance with ethical guidelines}

\section{Competing interests}

We have submitted a Chinese patent application on parts of this study (BrkAutoDisplay) with the application number 201310750736.6.

Received: 16 April 2015 Accepted: 10 August 2015 Published online: 04 September 2015

\section{References}

1. Schumacher SD, Hannemann F, Teese MG, Bernhardt R, Jose J (2012) Autodisplay of functional CYP106A2 in Escherichia coli. J Biotechnol 161:104-112

2. Kranen E, Detzel C, Weber T, Jose J (2014) Autodisplay for the co-expression of lipase and foldase on the surface of E. coli: washing with designer bugs. Microb Cell Fact 13:19

3. Fitting J, Blume T, Ten Haaf A, Blau W, Gattenlohner S, Tur MK, Barth S (2015) Phage display-based generation of novel internalizing antibody fragments for immunotoxin-based treatment of acute myeloid leukemia. MAbs 7:390-402

4. Brekke OH, Loset GA (2003) New technologies in therapeutic antibody development. Curr Opin Pharmacol 3:544-550

5. Lofblom J (2011) Bacterial display in combinatorial protein engineering. Biotechnol J 6:1115-1129

6. Harvey BR, Georgiou G, Hayhurst A, Jeong KJ, Iverson BL, Rogers GK (2004) Anchored periplasmic expression, a versatile technology for the isolation of high-affinity antibodies from Escherichia coli-expressed libraries. Proc Natl Acad Sci USA 101:9193-9198

7. Mazor Y, Van Blarcom T, Mabry R, Iverson BL, Georgiou G (2007) Isolation of engineered, full-length antibodies from libraries expressed in Escherichia coli. Nat Biotechnol 25:563-565

8. Jose J (2006) Autodisplay: efficient bacterial surface display of recombinant proteins. Appl Microbiol Biotechnol 69:607-614

9. Nicolay T, Vanderleyden J, Spaepen S (2015) Autotransporter-based cell surface display in Gram-negative bacteria. Crit Rev Microbiol 41:109-123

10. Daugherty PS (2007) Protein engineering with bacterial display. Curr Opin Struct Biol 17:474-480

11. Jose J, Maas RM, Teese MG (2012) Autodisplay of enzymes-molecular basis and perspectives. J Biotechnol 161:92-103

12. Verhoeven GS, Alexeeva S, Dogterom M, den Blaauwen T (2009) Differential bacterial surface display of peptides by the transmembrane domain of OmpA. PLoS One 4:e6739

13. Bassi AS, Ding DN, Gloor GB, Margaritis A (2000) Expression of single chain antibodies (ScFvs) for c-myc oncoprotein in recombinant Escherichia coli membranes by using the ice-nucleation protein of Pseudomonas syringae. Biotechnol Prog 16:557-563

14. Henderson IR, Navarro-Garcia F, Nataro JP (1998) The great escape: structure and function of the autotransporter proteins. Trends Microbiol 6:370-378

15. Dautin N, Bernstein HD (2007) Protein secretion in gram-negative bacteria via the autotransporter pathway. Annu Rev Microbiol 61:89-112

16. Benz I, Schmidt MA (2011) Structures and functions of autotransporter proteins in microbial pathogens. Int J Med Microbiol 301:461-468

17. Pohlner J, Halter R, Beyreuther K, Meyer TF (1987) Gene structure and extracellular secretion of Neisseria gonorrhoeae lgA protease. Nature 325:458-462

18. Sherlock O, Dobrindt U, Jensen JB, Munk Vejborg R, Klemm P (2006) Glycosylation of the self-recognizing Escherichia coli Ag43 autotransporter protein. J Bacteriol 188:1798-1807

19. Benz I, Schmidt MA (1992) AIDA-I, the adhesin involved in diffuse adherence of the diarrhoeagenic Escherichia coli strain $2787(\mathrm{O} 126: \mathrm{H} 27)$, is synthesized via a precursor molecule. Mol Microbiol 6:1539-1546

20. Ruiz-Olvera P, Ruiz-Perez F, Sepulveda NV, Santiago-Machuca A, Maldonado-Rodriguez R, Garcia-Elorriaga G, Gonzalez-Bonilla C (2003) Display and release of the Plasmodium falciparum circumsporozoite protein using the autotransporter MisL of Salmonella enterica. Plasmid $50: 12-27$

21. Webb CT, Selkrig J, Perry AJ, Noinaj N, Buchanan SK, Lithgow T (2012) Dynamic association of BAM complex modules includes surface exposure of the lipoprotein BamC. J Mol Biol 422:545-555

22. Leyton DL, Rossiter $A E$, Henderson IR (2012) From self sufficiency to dependence: mechanisms and factors important for autotransporter biogenesis. Nat Rev Microbiol 10:213-225

23. Leyton DL, Johnson MD, Thapa R, Huysmans GH, Dunstan RA, Celik N, Shen HH, Loo D, Belousoff MJ, Purcell AW et al (2014) A mortise-tenon joint in the transmembrane domain modulates autotransporter assembly into bacterial outer membranes. Nat Commun 5:4239

24. Roman-Hernandez G, Peterson JH, Bernstein HD (2014) Reconstitution of bacterial autotransporter assembly using purified components. Elife 3:e04234

25. Junker M, Besingi RN, Clark PL (2009) Vectorial transport and folding of an autotransporter virulence protein during outer membrane secretion. Mol Microbiol 71:1323-1332

26. Skillman KM, Barnard TJ, Peterson JH, Ghirlando R, Bernstein HD (2005) Efficient secretion of a folded protein domain by a monomeric bacterial autotransporter. Mol Microbiol 58:945-958

27. Dautin N, Barnard TJ, Anderson DE, Bernstein HD (2007) Cleavage of a bacterial autotransporter by an evolutionarily convergent autocatalytic mechanism. EMBO J 26:1942-1952

28. Nicolay T, Lemoine L, Lievens E, Balzarini S, Vanderleyden J, Spaepen S (2012) Probing the applicability of autotransporter based surface display with the EstA autotransporter of Pseudomonas stutzeri A15. Microb Cell Fact 11:158

29. Fernandez RC, Weiss AA (1994) Cloning and sequencing of a Bordetella pertussis serum resistance locus. Infect Immun 62:4727-4738

30. Zhai Y, Zhang K, Huo Y, Zhu Y, Zhou Q, Lu J, Black I, Pang X, Roszak AW, Zhang $X$ et al (2011) Autotransporter passenger domain secretion requires a hydrophobic cavity at the extracellular entrance of the betadomain pore. Biochem J 435:577-587

31. Oliver DC, Fernandez RC (2001) Antibodies to BrkA augment killing of Bordetella pertussis. Vaccine 20:235-241

32. Jain S, van Ulsen P, Benz I, Schmidt MA, Fernandez R, Tommassen J, Goldberg MB (2006) Polar localization of the autotransporter family of large bacterial virulence proteins. J Bacteriol 188:4841-4850

33. leva R, Skillman KM, Bernstein HD (2008) Incorporation of a polypeptide segment into the beta-domain pore during the assembly of a bacterial autotransporter. Mol Microbiol 67:188-201

34. Hullo MF, Moszer I, Danchin A, Martin-Verstraete I (2001) CotA of Bacillus subtilis is a copper-dependent laccase. J Bacteriol 183:5426-5430

35. Agnihotri G, Liu HW (2003) Enoyl-CoA hydratase. reaction, mechanism, and inhibition. Bioorg Med Chem 11:9-20

36. Bradshaw RA, Noyes BE (1975) L-3-hydroxyacyl coenzyme A dehydrogenase from pig heart muscle. EC 1.1.1.35 L-3-hydroxyacyl-CoA: NAD oxidoreductase. Methods Enzymol 35:122-128 
37. Botta B, Delle Monache G, Menendez P, Boffi A (2005) Novel prenyltransferase enzymes as a tool for flavonoid prenylation. Trends Pharmacol Sci 26:606-608

38. Petrovskaya LE, Novototskaya-Vlasova KA, Kryukova EA, Rivkina EM, Dolgikh DA, Kirpichnikov MP (2015) Cell surface display of cold-active esterase EstPc with the use of a new autotransporter from Psychrobacter cryohalolentis K5(T). Extremophiles 19:161-170

39. Lee HW, Byun SM (2003) The pore size of the autotransporter domain is critical for the active translocation of the passenger domain. Biochem Biophys Res Commun 307:820-825

40. Souriau C, Hudson PJ (2003) Recombinant antibodies for cancer diagnosis and therapy. Expert Opin Biol Ther 3:305-318
41. Xiao Y (2013) Comparison of autotransporter and ice nucleation proteinas carrier proteins for antibody display on the cell surface of Escherichia coli. Progress Biochem Biophys 40:1209-1219

42. Huang FY, Wang CC, Huang YH, Zhao HG, Guo JL, Zhou SL, Wang H, Lin YY, Tan GH (2014) Antigen 43/Fcepsilon3 chimeric protein expressed by a novel bacterial surface expression system as an effective asthma vaccine. Immunology 143:230-240

43. Kjaergaard K, Hasman H, Schembri MA, Klemm P (2002) Antigen 43-mediated autotransporter display, a versatile bacterial cell surface presentation system. J Bacteriol 184:4197-4204

\section{Submit your next manuscript to BioMed Central} and take full advantage of:

- Convenient online submission

- Thorough peer review

- No space constraints or color figure charges

- Immediate publication on acceptance

- Inclusion in PubMed, CAS, Scopus and Google Scholar

- Research which is freely available for redistribution

Submit your manuscript at 